\title{
A INFLUENCIA DA PANDEMIA DE COVID-19 NOS ATENDIMENTOS ODONTOLÓGICOS NA REGIÃO COREDES SERRA, HORTÊNSIAS E LITORAL DO RS
}

\author{
LA INFLUENCIA DE LA PANDEMIA COVID-19 EN LA ATENCIÓN DENTAL EN \\ COREDES SERRA, HORTÊNSIAS Y REGIÓN COSTERA DE RS
}
THE INFLUENCE OF THE COVID-19 PANDEMIC ON DENTAL CARE IN THE COREDES SERRA, HORTÊNSIAS AND COASTAL REGION OF RS

\author{
Deise Renata BRINGMANN ${ }^{1}$ \\ Maria Carolina Rosa GULLO ${ }^{2}$ \\ Thiago de OLIVEIRA GAMBA ${ }^{3}$
}

RESUMO: Com objetivo de investigar a influência que as restrições impostas pela pandemia da COVID-19 no ano de 2020 acarretaram ao fluxo de atendimento diário das clínicas odontológicas dos Conselhos Regionais de Desenvolvimento - COREDEs - Serra, Hortênsias e Litoral do Rio Grande do Sul-RS, realizou-se uma pesquisa quantitativa e exploratória. A pesquisa contou com a participação de 93 clínicas odontológicas, que responderam ao questionário utilizado. Os dados coletados revelaram que o isolamento social/profissional durante o período pandêmico de 2020, não só influenciou na sustentabilidade econômica das clínicas, como também afetou nas emoções destes profissionais. Esses relataram que, o risco de contágio e a preocupação de contaminar os familiares, deixava-os apreensivos. Portanto, conclui-se que houve um impacto socioeconômico e psicológico, devido a um somatório de acontecimentos gerados a partir da pandemia COVID-19.

PALAVRAS-CHAVE: Pandemia. Clínicas odontológicas. Prevenção à COVID-19. Impacto socioeconômico. Impacto psicológico.

RESUMEN: Con el objetivo de investigar la influencia que las restricciones impuestas por la pandemia de COVID-19 en el año 2020 trajeron al flujo de asistencia diaria de las clínicas dentales de los Consejos Regionales de Desarrollo - COREDEs - Serra, Hortênsias y Litoral de Rio Grande do Sul-RS, se realizó una investigación cuantitativa y exploratoria. La investigación contó con la participación de 93 clínicas dentales, que respondieron al cuestionario utilizado. Los datos recogidos revelaron que el aislamiento social/profesional durante el periodo de pandemia de 2020, no sólo influyó en la sostenibilidad económica de las clínicas, sino que también afectó a las emociones de estos profesionales. Estos informaron que, el riesgo de contagio y la preocupación de contaminar a los miembros de la familia, les

1 Universidade de Caxias do Sul (UCS), Caxias do Sul - RS - Brasil. Doutoranda no Programa de PósGraduação em Ciências da Saúde. ORCID: https://orcid.org/0000-0002-2849-225X. E-mail: deise.renata@ucs.br ${ }^{2}$ Universidade de Caxias do Sul (UCS), Caxias do Sul - RS - Brasil. Professora Adjunta III no Departamento de Ciências Sociais. Doutorado em Economia (UFRGS). ORCID: https://orcid.org/0000-0002-3835-8222. E-mail: mcrgullo@ucs.br

${ }^{3}$ Universidade de Caxias do Sul (UCS), Caxias do Sul - RS - Brasil. Professor do Curso de Odontologia. Doutorado em Radiologia Odontológica (UNICAMP). ORCID: https://orcid.org/0000-0002-3941-9672. E-mail: togamba@ucs.br 
hacía ser aprensivos. Por lo tanto, se concluye que hubo un impacto socioeconómico y psicológico, debido a una suma de eventos generados a partir de la pandemia de COVID-19.

PALABRAS CLAVE: Pandemia. Clínicas dentales. Prevención de COVID-19. Impacto socioeconómico. Impacto psicológico.

ABSTRACT: Aiming to investigate the influence that the restrictions imposed by the COVID19 pandemic in the year 2020 brought to the daily attendance flow of dental clinics of the Regional Development Councils - COREDEs - Serra, Hortênsias and Litoral of Rio Grande do Sul-RS, a quantitative and exploratory research was conducted. The research counted on the participation of 93 dental clinics, who answered the questionnaire used. The data collected revealed that the social/professional isolation during the pandemic period of 2020 not only influenced the economic sustainability of the clinics, but also affected the emotions of these professionals. These reported that, the risk of contagion and the concern of contaminating family members, made them apprehensive. Therefore, it is concluded that there was a socioeconomic and psychological impact, due to a sum of events generated from the COVID-19 pandemic.

KEYWORDS: Pandemic. Dental clinics. COVID-19 prevention. Socioeconomic impact. Psychological impact.

\section{Introdução}

O surgimento da SARS-coV-2, agente da doença COVID-19, em Wuhan na China em 2019, desencadeou uma pandemia global, que se estende até os dias atuais. No território brasileiro, a área da saúde adotou estratégias emergenciais para o combate ao contágio da COVID-19, tendo como principal foco a atenção primária à saúde dos brasileiros. Dentre as orientações estava a suspensão dos atendimentos odontológicos eletivos, sendo mantidos apenas os procedimentos de urgências e de emergência, com o objetivo de diminuir a probabilidade de contaminação cruzada (BRASIL, 2020a; BRASIL, 2020b).

$\mathrm{Na}$ odontologia uma preocupação latente em tempos de pandemia envolve o fato de que durante os procedimentos odontológicos há uma acentuada produção de gotículas e aerossóis, o que demanda medidas de biossegurança nas clínicas odontológicas, contudo não sendo um absoluto impeditivo na transmissibilidade do COVID-19. Esse aspecto está, segundo Meng, Hua e Bian (2020), em especial associada aos pacientes assintomáticos, haja vista que esses representam potenciais transmissores durante procedimentos odontológicos.

Neste horizonte, os cirurgiões-dentistas desempenharam funções essenciais voltadas para à execução de ações de controle da COVID-19. Uma vez que, os cirurgiões dentistas estão dentre os profissionais atuantes na linha de frente do enfrentamento ao vírus, atuando 
em ações de prevenção de doenças não restritas somente aos cuidados bucais. Desta forma, como mecanismo de prevenção e de controle da COVID-19, tem sido preconizado que os cirurgiões-dentistas reforcem as medidas de segurança por meio dos equipamentos de proteção individual (EPIs) e evitem, quando possível, a realização de procedimentos envolvendo aerossóis e de gotículas (AMIB/CFO,2020). Além de manter uma constante manutenção dos ambientes clínicos, mantendo uma rigorosa assepsia, de forma a dispor de ambientes limpos e secos, com vistas a não propagar o vírus da COVID-19.

Diante do exposto, averiguou-se quais foram os impactos que as restrições impostas pelo Ministério da Saúde (BRASIL, 2020) e pelo Conselho Federal de Odontologia-CFO (2020) acarretaram ao fluxo de atendimento diário das clínicas odontológicas que fazem parte dos Conselhos Regionais de Desenvolvimento (COREDEs) - Serra, Hortênsias e Litoral do Rio Grande do Sul (RS).

\section{A influência da pandemia no atendimento odontológico}

As restrições impostas pelos órgãos da saúde provocaram não só reflexos na economia, mas também trouxeram/trazem efeitos psicológicos, sendo que se tornou indispensável buscar meios de manter os cuidados com a saúde mental (OSÓRIO et al., 2021). Como sugere Osório et al. (2021), a incerteza, mais o sentimento ligado às consequências sociais e econômicas que aparentemente precedem o isolamento para a vida cotidiana, social e profissional. Uma vez que, algumas pessoas expressam saudade da família, afirmando que sua economia está afetada porque perderam o emprego, outras relatam que se sentem angustiadas pelos familiares que devem comparecer ao trabalho, tristeza pelos mais vulneráveis, preocupação com as finanças familiares. Levando em consideração que, o vírus é altamente transmissível, de forma que as interações representam risco de contaminação grave em 19\% dos casos (KANG et al., 2020), e que a eficácia dos protocolos de manejo clínico é incerta (PFEFFERBAUM; NORTH, 2020), as medidas preventivas e rígidas tornaram-se necessárias e indispensáveis durante a pandemia da COVID-19.

Pesquisas realizadas em vários países revelam que os profissionais de saúde que trabalham na linha de frente contra o COVID-19 têm experimentado as taxas mais altas de transtornos mentais. A meta-análise conduzida por De Pablo et al. (2020) constatou que 63\% dos trabalhadores têm uma preocupação geral com sua saúde, $44 \%$ temem o contágio e 38\% experimentam insônia. Para Conti et al. (2020) os principais impactos estariam relacionados à insegurança, ao estado de alerta constante, aos sintomas depressivos como, tristeza, solidão, 
perda da qualidade do sono e desmotivação, bem como maior instabilidade emocional. De acordo com Gameiro (2020), estima-se que $30 \%$ a $50 \%$ da população pode sofrer alguma manifestação psicopatológica, se nenhum cuidado for promovido. Segundo Osório et al. (2021) "o estresse é fator de risco para vários transtornos mentais", por outro lado, "o pânico pode ser disparado nos casos de maior ansiedade", afirma Mari (2020) médico psiquiatra. Esses aspectos emocionais são, geralmente, pontos centrais a serem considerados em face de uma pandemia.

A COVID-19 também trouxe sérios riscos aos cirurgiões-dentistas, na medida em que gerou o afastamento desses profissionais de sua rotina de trabalho, em uma variável de entre 01 e 40 dias, segundo Gomes et al. (2020). Essa informação explica o agravamento sistêmico da COVID-19 no Brasil. A pesquisa de Gomes et al. (2020) ainda aponta dados referentes à diminuição de consultas odontológicos, e, consequentemente a queda de faturamento, ocasionada pelo cenário de crise econômica brasileira. Entretanto, notou-se uma representativa disparidade econômica nos diferentes Estados do Brasil (CHAMORRO et al., 2020; PASSARELLI et al., 2020). A pandemia COVID-19 não causou apenas um impacto em nível de saúde pública, mas também uma grande recessão econômica mundial, o que gerou perdas de empregos e continua a ameaçar fortemente a economia global, as sociedades e os direitos humanos (ONU, 2020).

Neste horizonte, o presente artigo investiga a influência da COVID-19 no fluxo de atendimento das clínicas odontológicas, que fazem parte dos Conselhos Regionais de Desenvolvimento (COREDEs) - Serra, Hortênsias e Litoral do Rio Grande do Sul (RS). Uma vez que as clínicas odontológicas atendem diretamente ao público, infere-se que diante de uma crise econômica essas podem ter adotado estratégias sustentáveis, de maneira a se manterem no mercado, sendo esse o objetivo de estudo deste artigo. Para tanto, o estudo volta-se a aspectos como: quantidade de consultas; percentual de consultas de atenção primária anterior ao período pandêmico e percentual de consultas primárias durante o período pandêmico de 2020; quantas consultas de atenção especializada eram realizadas por dia na clínica antes da pandemia; e percentual de consultas especializadas durante o período pandêmico de 2020 e o motivo dos cancelamentos e reagendamentos.

De modo a aprofundar o tema aqui apresentado, esta pesquisa apoia-se na literatura sobre pandemia (GULLO, 2020; LIU, KUO, SHIH, 2020; SENHORAS, 2020; XAVIER et al., 2020), sustentabilidade na área da saúde (AVINASH et al., 2013; FEIL; MULIMANI, 2017; SCHREIBER, 2017) e sustentabilidade relacionada ao campo da odontologia 
(ARAÚJO, 2010; GLICK, 2016; MORITA; HADDAD, 2020). A seguir, apresenta-se o método de pesquisa adotado para fins de operacionalização do fazer científico aqui proposto.

\section{Material e método}

Esta é uma pesquisa quantitativa exploratória (SAMPIERI; COLLADO; LUCIO, 2013) vinculada a uma pesquisa de doutorado na área da Saúde, relacionada à Odontologia, aprovada pelo Comitê de Ética em Pesquisa (CEP) da Universidade XXXX (revisão às cegas), sob o parecer de número CAAE: 39814720.9.0000.5341. O presente objeto de estudo deste artigo configura-se como um recorte da pesquisa, base supracitada, ao passo em que se centra, especificamente, no caráter de sustentabilidade socioeconômica informados pelas clínicas odontológicas antes e durante a pandemia da COVID-19. O questionário foi o instrumento de coleta de dados aplicado aos sócios-proprietários de clínicas odontológicas localizadas nos municípios que fazem parte dos Conselhos Regionais de Desenvolvimento COREDEs, Serra, Hortênsias e Litoral do Rio Grande do Sul $-\mathrm{RS}^{4}$. A investigação realizada focou-se em caráter quantitativo, com vistas a aproximar os dados obtidos da teoria e auxiliar na compreensão do problema de pesquisa. O que concerne ao percurso, na primeira fase, realizou-se uma breve apresentação das diretrizes de biossegurança postuladas pelos órgãos de saúde e, na segunda fase, tratou-se da coleta dos dados junto às clínicas odontológicas, ao passo em que na terceira e última fase, conduziu-se a análise dos resultados. Os dados coletados foram tabulados e classificados com base na aporte teórico-metodológico adotado nesta pesquisa.

Relativo à coleta de dados, referente ao fluxo de atendimento das clínicas odontológicas, essa deu-se por meio de um questionário semiestruturado, composto por 27 perguntas, a saber, 16 questões abertas e 11 questões fechadas. Para este estudo, fez-se um recorte investigativo a partir de 04 questões dissertativas, a saber, acerca de aspectos como: (a) número de consultas primárias antes da pandemia (b) número de consultas diárias de atenção primária durante a pandemia; (c) número de consultas diárias especializadas durante a pandemia; (d) número de consultas especializadas diárias antes da pandemia. As clínicas foram classificadas por números de 1 a 126, dentre as clínicas participantes 47 retornaram em branco.

\footnotetext{
${ }^{4}$ Municípios integrantes dos COREDEs que fizeram parte desta pesquisa: Caxias do Sul, Farroupilha, Bento Gonçalves, Veranópolis, Nova Prata, Carlos Barbosa (COREDE, Serra) Gramado, Canela, Nova Petrópolis (COREDE, Hortências), Torres, Capão da Canoa e Arroio do Sal (COREDE, Litoral).
} 
Diante do exposto, averiguou-se quais foram os impactos que as restrições impostas pelo Ministério da Saúde (BRASIL, 2020) e pelo Conselho Federal de Odontologia-CFO (2020) acarretaram ao fluxo de atendimento diário das clínicas odontológicas. Neste tocante, a pesquisa contou com a participação de 126 clínicas odontológicas que responderam ao instrumento de pesquisa. A aplicação do questionário ocorreu no período de 06 de janeiro a 31 de maio de 2021, de modo presencial, quando esses foram entregues e posteriormente coletados. O cálculo amostral foi feito a partir do software Statulator ${ }^{5}$. Quanto ao fator de interesse, tendo como tamanho populacional 220 respondentes, o estudo exigia um tamanho mínimo amostral de 140 respondentes. Em outras palavras, infere-se que, a partir de 140 respondentes, $50 \%$ destes tinham fator de interesse, o que representou $95 \%$ de fator confiança. No entanto, foram contabilizados apenas 93 questionários, uma vez que 47 questionários retornaram em branco.

Para a análise de dados utilizou-se o pacote da Microsoft planilhas do Excel, para fins de análise e tabulação dos dados coletados. Além disso, os dados lapidados foram organizados em gráficos. A análise de dados teve o aporte teórico-metodológico na área de sustentabilidade econômica (DE OLIVEIRA et al., 2020; GULLO, 2020; MILLER, 2019; NETO, 2020) da gestão econômica (FERRAZ, 2020; SCHWENDICKE; KROIS; GOMEZ, 2020). A seguir, apresenta-se os resultados da pesquisa.

\section{Resultados}

A aplicação de instrumento de coletas de dados ocorreu com a participação efetiva de 93 clínicas odontológicas, foram escolhidas aleatoriamente 12 clínicas por município. Cabe ressaltar, que, inicialmente, foram entregues 140 questionários dos quais, 14 não responderam o questionário e 33 não responderam às 04 questões dissertativas, totalizando a exclusão de 47 clínicas desta pesquisa. Os munícipios mencionados constituem os Conselhos Regionais de Desenvolvimento - COREDEs, Serra, Hortênsias e Litoral do Rio Grande do Sul -RS. Com o objetivo de preservar a identidade das clínicas participantes, elas foram numeradas de 1 a 126 e os dados foram registrados de acordo com o número designado.

De modo a organizar os dados obtidos e tratados nesta pesquisa, a seguir, apresenta-se o gráfico 1a,1b e1c, no qual consta o fluxo das consultas durante a pandemia no ano de 2020 nas clínicas odontológicas. Os dados apresentados foram distribuídos em três partes, de modo que fosse possível apresentar ao leitor uma visão panorâmica do todo do fluxo de consultas.

${ }^{5}$ Disponível em: https://statulator.com/SampleSize/ss1P.html. Acesso em: 20 jan. 2021. 


\section{Figura 1}

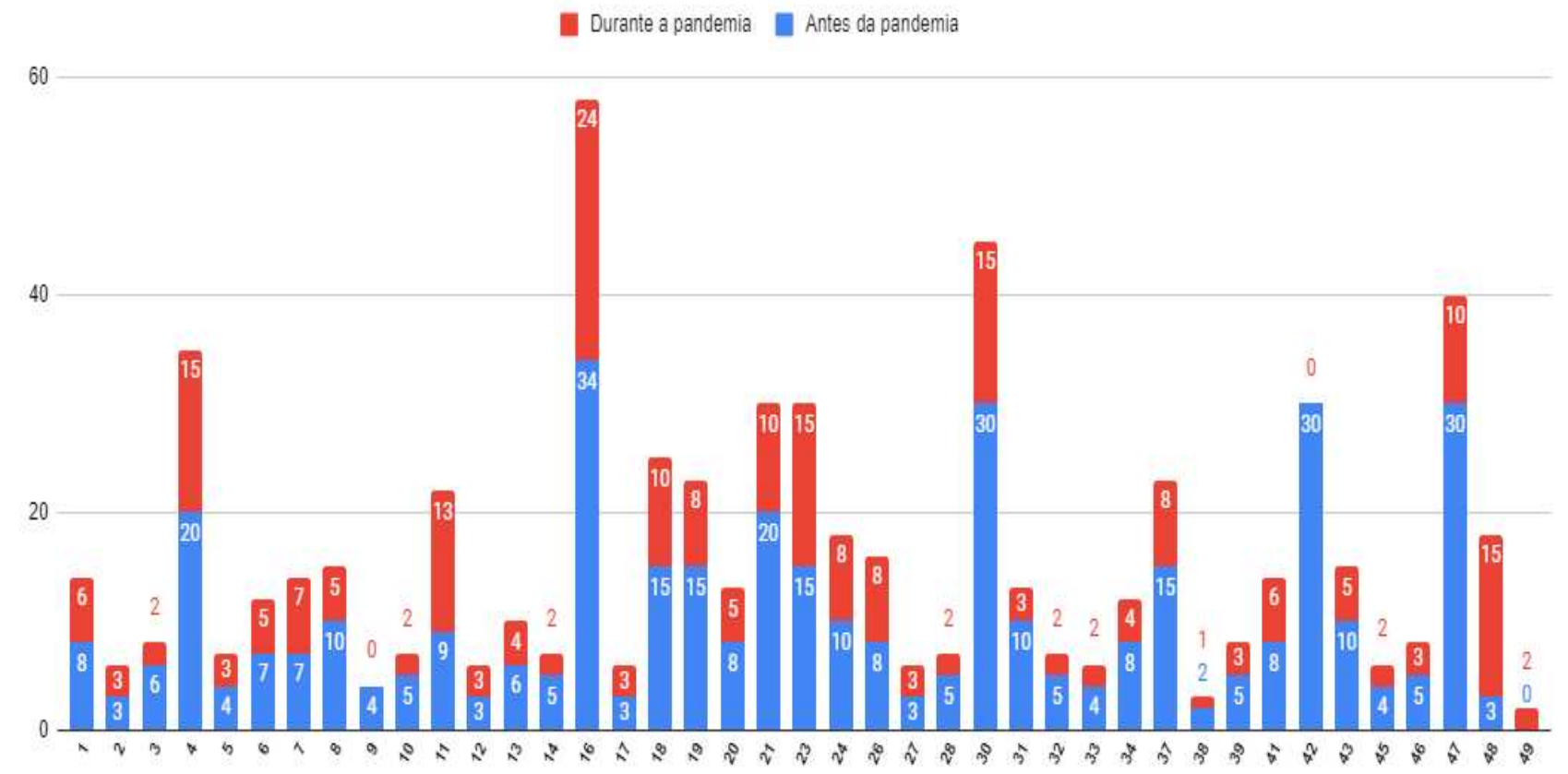

Fonte: Elaborado pelos autores

Figura 2

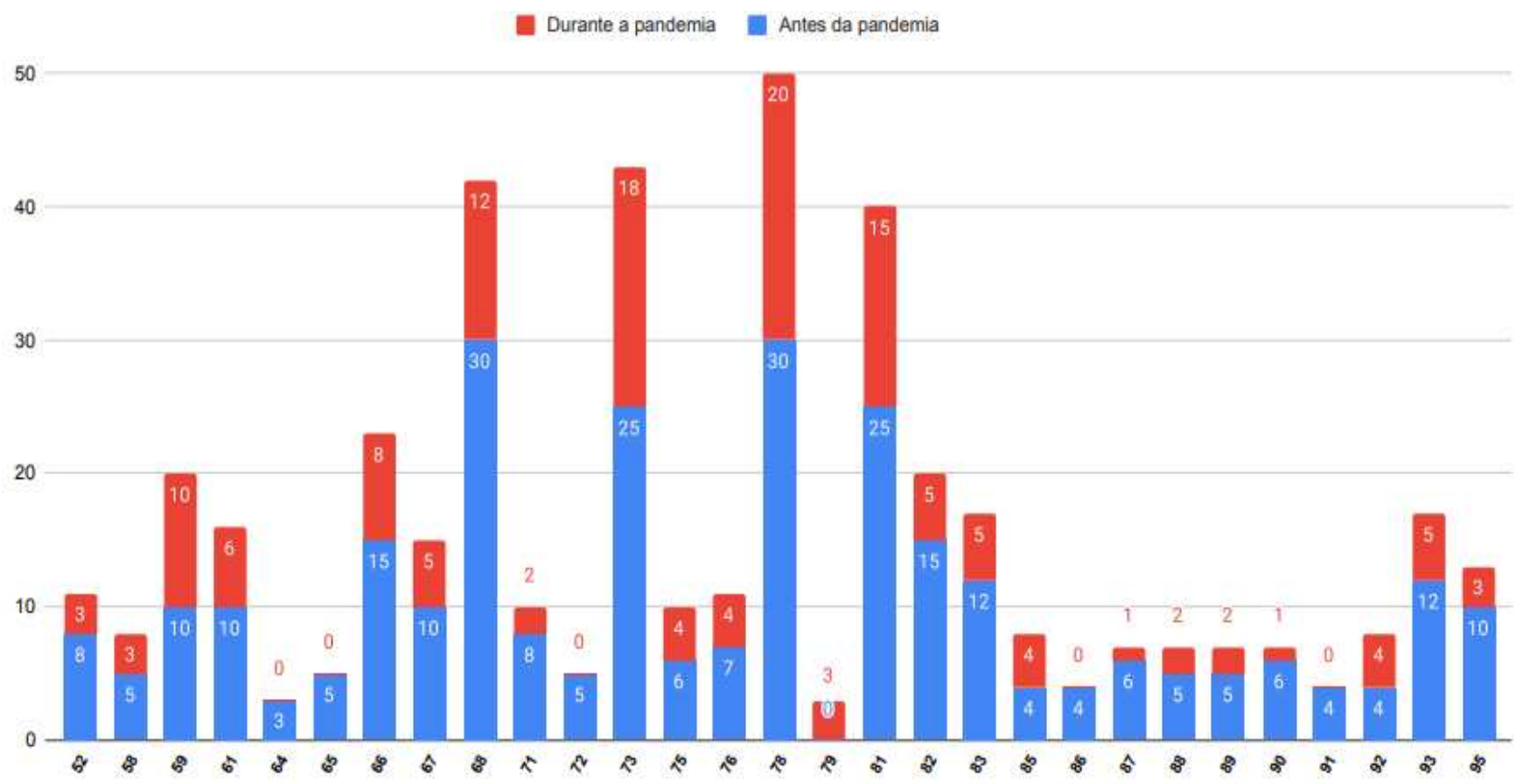

Fonte: Elaborado pelos autores 


\section{Figura 3}

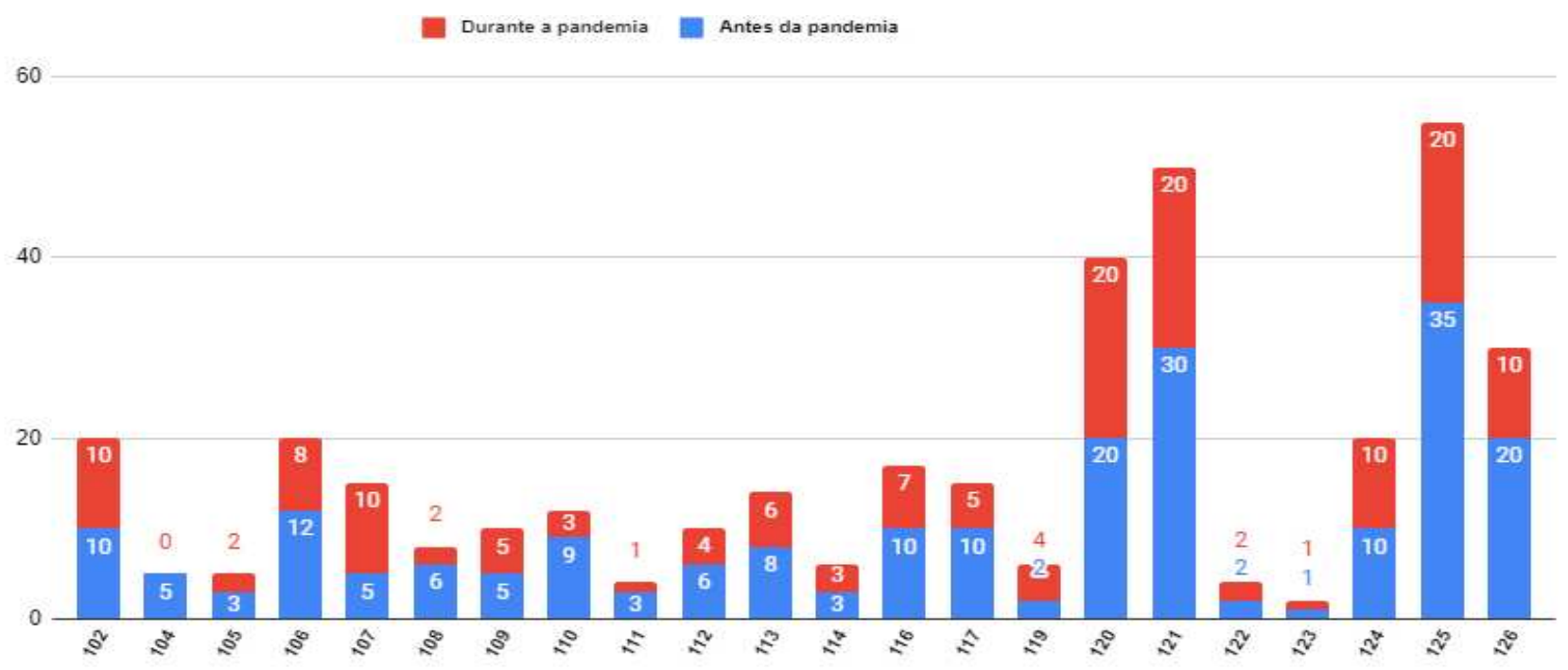

Fonte: Elaborado pelos autores

Com base nos dados, apresentados constatou-se que média de consultas de atenção primária antes do período pandêmico era de 10 consultas e durante foi de 5 (queda de $50 \%$ ) e nas consultas especializadas antes do período pandêmico era de 11 consultas e durante foi de 6 consultas na média (queda de 45\%). A partir dos cancelamentos os cirurgiões-dentistas tiveram redução na renda e aumento nas despesas do consultório. De acordo com relatos dos cirurgiões-dentistas os cancelamentos de consultas ocorreram nos seis (6) primeiros meses da pandemia COVID-19 no ano de 2020, o que revelou uma preocupação expressiva do ponto de vista psicológico dos pacientes quanto à possibilidade de contágio e transmissão desse para seus familiares, resultado esse também mencionado na pesquisa realizada por Osório et al., (2021). Os dados apontaram que as medidas preventivas que foram tomadas a fim de mitigar os impactos da pandemia, tendo como estratégia principal o lock-down (confinamento) em períodos e durações distintas, a depender da elevação de contaminação, provocou uma queda na atividade econômica, o que afetou a condição econômica dos pacientes e das clínicas odontológicas (BRASIL, 2020a; 2020b). Ou seja, as restrições impostas pelo Ministério da Saúde (BRASIL, 2020) e pelo CFO (2020) atingiram diretamente o fluxo de atendimento nas clínicas odontológicas que pode ter repercutido nos modos de conduzir a gestão da clínica.

Os cirurgiões-dentistas relataram que, houve escassez de procura por atendimento, médico-odontológico, redução de fornecedores de insumos, aumento de custo com equipamentos de proteção individual. Esses fatores desencadearam um impacto econômico e 
um possível desgaste emocional nos profissionais da odontologia durante a pandemia COVID-19.

\section{Considerações finais}

A Odontologia tem sido considerada uma das profissões de maior risco ocupacional, devido ao contato íntimo e à relação com saliva e sangue. Diante disso, fez-se indispensável adotar medidas preventivas muito rígidas e onerosas, assim como, inicialmente, atender apenas consultas de emergência.

Embora o distanciamento social seja considerado uma das principais estratégias para prevenir a transmissão viral durante a pandemia de COVID-19, elas acarretaram uma influência negativa na economia. A pesquisa apontou uma queda no atendimento odontológico durante a pandemia COVID-19, a média de atendimentos era de 10 consultas e durante foi de 5 (queda de 50\%) e nas consultas especializadas antes do período pandêmico era de 11 consultas e durante foi de 6 (queda de 45\%) consultas na média. De acordo com relatos dos cirurgiões-dentistas, os cancelamentos de consultas ocorreram nos seis (6) primeiros meses da pandemia COVID-19 no ano de 2020, o que revelou um expressivo abalo envolvimento pessoal e emocional dos pacientes neste contexto desafiador. $\mathrm{O}$ isolamento social e profissional durante o período pandêmico de 2020, não só influenciou a sustentabilidade econômica das clínicas, como também afetou as emoções destes profissionais, eles relataram que, o risco de contágio e a preocupação de contaminar os familiares, deixou-os em constante agonia. Dantas et al. (2020), postula que "a pandemia da COVID-19 já provocou um processo de luto coletivo na população pela perda da vida normal e deve agravar o luto vivido por familiares e amigos de quem morre por causa da Covid.”. Somado a isso, houve também as restrições impostas pela Agência Nacional de Vigilância Sanitária (ANVISA, 2020) recomendando que os atendimentos odontológicos eletivos fossem adiados, restringindo o atendimento clínico apenas aos casos de urgência e emergência. Complementarmente, a Associação de Medicina Intensiva Brasileira (AMIB), junto com o Conselho Federal de Odontologia (CFO), recomendou que pacientes com suspeita ou com COVID-19 necessitando de atendimento de urgência e emergência deviam ter o tratamento realizado com precauções de biossegurança para toda a equipe (AMIB/CFO, 2020). Vale ressaltar que estas recomendações sugeridas pela AMIB e CFO não têm poder de lei, mas embasam a tomada de decisão pelos gestores competentes. 
Para Da Silva et al. (2020), as medidas de proteção utilizadas antes da pandemia, não são totalmente eficazes para impedir a contaminação por COVID-19, causando apreensão nos profissionais e a necessidade de adquirirem conhecimento sobre as formas de prevenção e de controle da COVID-19. Conforme Pereira e Kemper (2020), a adoção de modificações na organização física dos ambulatórios e nas suas rotinas de atendimento, o rigor nos protocolos de biossegurança e o critério quanto à realização de tratamentos eletivos parecem ser a chave para o sucesso no enfrentamento dos novos desafios dos serviços odontológicos.

Para tanto, entendeu-se que a Odontologia sofreu influência por esse período pandêmico, entretanto, os antigos preceitos de biossegurança alicerçam as condutas a serem seguidas até que inovações técnicas possam ser comprovadas, cientificamente, e tornadas viáveis aos serviços odontológicos. Diante disso, conclui-se que houve um impacto econômico e psicológico generalizado devido a um somatório de acontecimentos proporcionados a partir da pandemia COVID-19.

Cabe destacar que a pesquisa de campo e a redação deste trabalho, foram conduzidas durante a pandemia, ou seja, a pandemia ainda está em curso, sendo assim, os dispositivos legais aqui abordados estão sendo constantemente atualizados e/ou modificados.

AGRADECIMENTOS: O presente trabalho foi realizado com apoio da Coordenação de Aperfeiçoamento de Pessoal de Nível Superior - Brasil (CAPES) - Código de Financiamento 001 .

\section{REFERÊNCIAS}

ANVISA. AGÊNCIA NACIONAL DE VIGILÂNCIA SANITÁRIA. Nota técnica GVIMS/GGTES/ANVISA n. 04/2020. Orientações para serviços de saúde: medidas de prevenção e controle que devem ser adotadas durante a assistência aos casos suspeitos ou confirmados de infecção pelo novo coronavírus (SARSCoV-2). Disponível em: https://www.gov.br/anvisa/pt-br/centraisdeconteudo/ publicacoes/servicosdesaude/notastecnicas/nota-tecnicagvims_ggtes_anvisa-04_2020-25-02-para-o-site.pdf. Acesso em: 21 ago. 2021

ABOL. ASSOCIAÇÃO BRASILEIRA DE ÉTICA E ODONTOLOGIA LEGAL. Orientações odontolegais com relação à retomada de atividades de ensino. Disponível em: https://docs.wixstatic.com/ugd/1adae6_3120b99a7698487ea2f2b8183efb400f.pdf. Acesso em: 10 jul. 2021.

AMIB/CFO. ASSOCIAÇÃO DE MEDICINA INTENSIVA BRASILEIRA e CONSELHO FEDERAL DE ODONTOLOGIA. Recomendações AMIB/ CFO para atendimento odontológico COVID-19. Disponível em: https://website.cfo.org.br/wp- 
content/uploads/2021/03/Recomendacoes-AMIB-CFO-para-enfrentamento-da-covid-19-naOdontologia.pdf Acesso em: 10 jul. 2021.

AVINASH, B. et al. Going green with eco-friendly dentistry. The Journal of Contemporary Dental Practice, v. 14, n. 4, p. 766-769, 2013.

BAO, Y. et al. 2019-nCoV epidemic: address mental health care to empower society. The Lancet, v. 395, n. 10224, p. 37-38, 2020.

BRASIL. Ministério da Saúde. Nota Técnica n. 9/2020-CGSB/DESF/SAPS/MS. COVID-19 e atendimento odontológico no SUS. 2020a. Disponível em: http://www.crosp.org.br/uploads/arquivo/295c9c14409db20cb63c862bb07ce0e4.pdf. Acesso em: 17 jul. 2021.

BRASIL. Secretaria de atenção primária à saúde. Protocolo de manejo clínico do coronavírus (COVID-19) na atenção primária à saúde. 2020b. Disponível em: https://www.gov.br/saude/pt-br/assuntos/noticias/ministerio-da-saude-lanca-orientacoes-parapadronizacao-do-atendimento-a-covid-19.pdf. Acesso em: 17 jul. 2021.

CONTI, C. et al. Fragile heroes. The psychological impact of the COVID-19 pandemic on health-care workers in Italy. PloS One, v. 15, n. 11, p. 1-17, 2020. DOI: doi.org/10.1371/journal.pone.0242538

DA SILVA, C. C. et al. Desafios da prevenção do novo coronavírus (COVID-19) na prática odontológica. Saúde Coletiva (Barueri), v. 10, n. 57, p. 3597-3606, 2020.

DE OLIVEIRA, J. J. M. et al. O impacto do coronavírus (covid-19) na prática odontológica: desafios e métodos de prevenção. Revista Eletrônica Acervo Saúde, v. 46, n. e3487, p.1-12, 2020. DOI: doi.org/10.25248/reas.e3487.2020

DE PABLO, G. S. et al. Impact of coronavirus syndromes on physical and mental health of health care workers: Systematic review and meta-analysis. Journal of Affective Disorders, v. 275 , p. $48-57,2020$.

FEIL, A. A.; SCHREIBER, D. Sustentabilidade e desenvolvimento sustentável: desvendando as sobreposições e alcances de seus significados. Cadernos Ebape BR, v. 15, n. 3, p. 667681, 2017.

FERRAZ, A. R. As grandes Pandemias da História. Revista De Ciência Elementar, v. 8, n. 2, p. 1-16, 2020. DOI: doi.org/10.24927/rce2020.025

FIOCRUZ. Saúde mental e atenção psicossocial na pandemia covid-19: recomendações gerais. Rio de Janeiro, RJ: Fiocruz, 2020. Disponível em:

https://www.fiocruzbrasilia.fiocruz.br/wp-content/uploads/2020/04/Sa\%C3\%BAde-Mental-eAten $\% \mathrm{C} 3 \% \mathrm{~A} 7 \% \mathrm{C} 3 \% \mathrm{~A} 30-P$ sicossocial-na-Pandemia-Covid-19-

recomenda\%C3\%A7\%C3\%B5es-gerais.pdf. Acesso em: 6 set. 2020.

GAMEIRO, N. Coronavírus e saúde mental. Brasília, DF: Fiocruz, 2020. Disponível em: https://www.fiocruzbrasilia.fiocruz.br/coronavirus-e-saude-mental-tire-suas-duvidas-aqui/. Acesso em: 21 ago. 2021 
GLICK, M. et al. A new definition for oral health developed by the FDI World Dental Federation opens the door to a universal definition of oral health. British Dental Journal, v. 221, n. 12, p. 792-793, 2016.

GOMES, P. et al. O Impacto do Coronavírus (COVID-19) as atividades odontológicas: desafios econômicos e mentais. Research, Society and Development, v. 10, n. 1, p. e22310111207-e22310111207, 2021.

GULLO, M. C. A economia na pandemia Covid-19: algumas considerações. ROSA DOS VENTOS-Turismo e Hospitalidade, v. 12, n. 3, 2020. DOI:

dx.doi.org/10.18226/21789061.v12i3a05

KANG LIJUN, Y. et al. La salud mental de los trabajadores médicos en Wuhan, China, lidiando con el nuevo coronavirus de 2019. The Lancet Psychiatry, v. 7, n. 3, p. 14, 2020.

LIU, Y.-C.; KUO, R.-L.; SHIH, S.-R. COVID-19: The first documented coronavirus pandemic in history. Biomedical Journal, v. 43, n. 4, p. 328-333, 2020.

MARI, J. O impacto da pandemia do COVID-19 na Sáude Mental. São Paulo, 2020. Disponível em: https://unifesp.br/noticias-anteriores/item/4395-quais-os-principais-efeitosdapandemia-na-saude-menta. Acesso em: 21 ago. 2021

MENG, L.; HUA, F.; BIAN, Z. Coronavirus disease 2019 (COVID-19): emerging and future challenges for dental and oral medicine. Journal of Dental Research, v. 99, n. 5, p. 481-487, 2020 .

MILLER, A. R. Perspectivas no gerenciamento da Covid-19 no atendimento odontológico. 2021. Dissertação (Mestrado Profissional em Administração - Gestão em Sistemas de Saúde) - UNINOVE, São Paulo, 2021.

MORITTA, M. C.; HADDAD, A. E.; ARAÚJO, M. E. Perfil atual e tendências do cirurgiãodentista brasileiro. In: Perfil atual e tendências do cirurgião-dentista brasileiro. 2010.

MULIMANI, P. Green dentistry: the art and science of sustainable practice. British Dental Jornal, v. 222, n. 12, p. 954-961, 2017.

NETO, R. B. G. Impactos da covid-19 sobre a economia mundial. Boletim de Conjuntura (BOCA), v. 2, n. 5, p. 113-127, 2020. DOI: dx.doi.org/10.5281/zenodo.3786698

ONU. ORGANIZAÇÃO DAS NAÇÕES UNIDAS. COVID-19: apelo urgente para uma resposta à recessão econômica dos direitos humanos. Especialista Independente da ONU. Genebra: ONU, 2020. Disponível em: https://www.hrw.org/pt/news/2020/03/23/339654. Acesso em: 21 ago. 2021.

OSÓRIO, F. L. et al. Risk and Protective Factors for the Mental Health of Brazilian Healthcare Workers in the Frontline of COVID-19 Pandemic. Frontiers in Psychiatry, v. 12, n. 662742, p. 1-9, 2021. 
PFEFFERBAUM, B.; NORTH, C. S. Mental health and the Covid-19 pandemic. New England Journal of Medicine, v. 383, n. 6, p. 510-512, 2020.

SAMPIERI, R. H.; COLLADO, C. F.; LUCIO, M. P. B. Metodologia de pesquisa. 5. ed. Porto Alegre, RS: Penso, 2013.

SCHWENDICKE, F.; KROIS, J.; GOMEZ, J. Impact of SARS-CoV2 (Covid-19) on dental practices: Economic analysis. Journal of Dentistry, v. 99, n. 103387, p. 1-6, 2020.

SENHORAS, E. M. COVID-19: enfoques preventivos. Boa Vista, RR: EdUFRR, 2020.

SILVA, R. F. et al. Proposta de inquéritos administrativo e clínico para tomada de decisão no atendimento odontológico em tempos de pandemia de covid-19. Revista Brasileira de Odontologia Legal, v. 7, n. 3, p. 41-54, 2020.

WU, K. Y. et al. COVID-19's impact on private practice and academic dentistry in North America. Oral Diseases, v. 27, n. 3 p. 684-687, 2021.

XAVIER, J. et al. A atuação do cirurgião-dentista, vinculado a um programa de residência multiprofissional em saúde, no combate à COVID-19 na Atenção Primária à Saúde. JMPHC Journal of Management \& Primary Health Care, v. 12, p. 1-16, 2020.

\section{Como referenciar este artigo}

BRINGMANN, D. R.; GULLO, M. C. R.; OLIVEIRA GAMBA, T. A influência da pandemia de COVID-19 nos atendimentos odontológicos na região Coredes Serra, Hortênsias e Litoral do RS. Doxa: Rev. Bras. Psico. E Educ., Araraquara, v. 22, n. 00, e021011, 2021. e-ISSN: 2594-8385. DOI: https://doi.org/10.30715/doxa.v22i00.15674

Submetido em: $27 / 07 / 2021$

Revisões requeridas em: 15/08/2021

Aprovado em: 20/09/2021

Publicado em: 01/10/2021 